\title{
On-axis joint transform correlation based on a four-level power spectrum
}

\author{
Ignasi Labastida, Arturo Carnicer, Estela Martín-Badosa, Ignacio Juvells, and \\ Santiago Vallmitjana
}

\begin{abstract}
We propose a method to obtain a single centered correlation with use of a joint transform correlator. We analyze the required setup to carry out the whole process optically, and we also present experimental results. () 1999 Optical Society of America

OCIS codes: $\quad 070.5010,070.4550,230.3720$.
\end{abstract}

\section{Introduction}

The joint transform correlator (JTC) ${ }^{1}$ has been shown to be a powerful coherent processor for optical pattern recognition. Several improvements in this architecture have been proposed to obtain real-time detection by use of liquid-crystal displays (LCD's).2,3 Most of the papers published in the pattern recognition field have analyzed different systems to increase the discrimination capability. A remaining drawback of JTC's is that multiple terms appear at the correlation plane, owing to the spatial separation of the scene and the reference at the input plane. A solution to this problem is based on focusing each diffracted term on a different plane. ${ }^{4}$ Nevertheless, the problem is not well solved, because the nonfocused terms overlap with the focused terms. Recently, some authors 5,6 have proposed the use of phase-shifting methods to get a zero-order diffraction term in a JTC, whereas other authors ${ }^{7}$ have presented a method of removing the zero-order spectrum to improve the performance of the JTC. In this study we propose an approach for obtaining a single centered correlation. This can be achieved by introduction of the scene and the reference superimposed at the input plane.

\section{Joint Transform Correlator Review}

A simple scheme of the JTC architecture is shown in Fig. 1. The scene and the reference are jointly displayed on an input LCD:

$$
f_{R}\left(x+x_{0} / 2, y+y_{0} / 2\right)+f_{S}\left(x-x_{0} / 2, y-y_{0} / 2\right) .
$$

The authors are with the Departament de Física Aplicada i Òptica, Universitat de Barcelona, Av. Diagonal 647, E08028 Barcelona, Spain. I. Labastida's e-mail address is nasi@optica.ub.es.

Received 18 November 1998; revised manuscript received 15 June 1999.

0003-6935/99/296111-05\$15.00/0

(C) 1999 Optical Society of America
We assume that the scene is located at $\left(x_{0} / 2, y_{0} / 2\right)$ and that the reference is at $\left(-x_{0} / 2,-y_{0} / 2\right)$. The intensity of the optical Fourier transform performed by a lens system is registered by a CCD camera. This distribution is called the joint power spectrum (JPS), and it is described by

$$
\begin{aligned}
I(u, v)= & \left|F_{R}(u, v)\right|^{2}+\left|F_{S}(u, v)\right|^{2} \\
& +2\left|F_{R}(u, v)\right|\left|F_{S}(u, v)\right| \cos \left[2 \pi\left(x_{0} u+y_{0} v\right)\right. \\
& \left.+\phi_{R}(u, v)-\phi_{S}(u, v)\right]
\end{aligned}
$$

where $\left|F_{R}(u, v)\right| \exp \left[i \phi_{R}(u, v)\right]$ and $\left|F_{S}(u, v)\right| \exp \left[i \phi_{S}(u\right.$, $v)]$ are the Fourier transforms of the reference $f_{R}(x, y)$ and the scene $f_{S}(x, y)$, respectively. In turn, the JPS is subsequently displayed on the LCD; so a second Fourier transformation $(\mathscr{F})$ gives

$$
\begin{aligned}
c(x, y)= & \mathscr{F}\{I(u, v)\} \\
= & f_{R}(x, y) \otimes f_{R}(x, y)+f_{S}(x, y) \otimes f_{S}(x, y) \\
& +f_{S}(x, y) \otimes f_{R}(x, y) * \delta\left(x-x_{0}, y-y_{0}\right) \\
& +f_{R}(x, y) \otimes f_{S}(x, y) * \delta\left(x+x_{0}, y+y_{0}\right),
\end{aligned}
$$

where the symbols $\otimes$ and $*$ stand for the correlation and the convolution product, respectively. Two cross-correlation terms appear centered at points $\left(x_{0}\right.$, $\left.y_{0}\right)$ and $\left(-x_{0},-y_{0}\right)$. The other two terms give nonuseful information, because they are self-correlation products. To avoid overlapping between terms, the scene and the reference at the input plane must be sufficiently far from each other.

The binarization of the JPS has been widely used as a way to improve the discrimination capability of the recognition system. ${ }^{8}$ We obtain the binary JPS, 


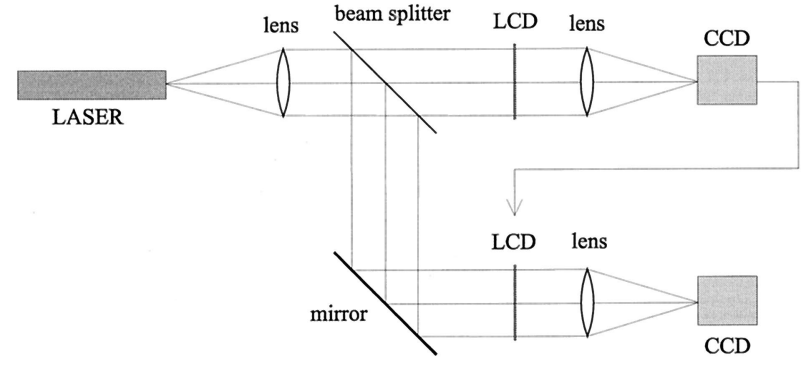

Fig. 1. Classic JTC.

$I_{b}(u, v)$, by assigning the values +1 or -1 to $I(u, v)$, according to the equation

$$
I_{b}(u, v)=B[I(u, v)]=\left\{\begin{array}{cl}
1 & \text { if } I(u, v) \geq I_{T}(u, v) \\
-1 & \text { if } I(u, v)<I_{T}(u, v)
\end{array},\right.
$$

where $B[]$ is the binarization operator and $I_{T}(u, v)$ is a predetermined threshold. This bipolar function can be expressed as a Fourier expansion ${ }^{8}$ :

$$
\begin{aligned}
I_{b}(u, v)= & \sum_{n=1}^{\infty} A_{n}\left[u, v ; I_{T}(u, v)\right] \cos \left\{n \left[2 \pi\left(x_{0} u+y_{0} v\right)\right.\right. \\
& \left.\left.+\phi_{R}(u, v)-\phi_{S}(u, v)\right]\right\},
\end{aligned}
$$

where

$$
\begin{aligned}
& A_{n}\left[u, v ; I_{T}(u, v)\right]= \\
& \quad \frac{4}{\pi n} \sin \left\{n \cos ^{-1}\left[\frac{\left|F_{R}(u, v)\right|^{2}+\left|F_{S}(u, v)\right|^{2}-I_{T}(u, v)}{2\left|F_{R}(u, v)\right|\left|F_{S}(u, v)\right|}\right]\right\} .
\end{aligned}
$$

The term that generates the first diffracted order is

$$
\begin{aligned}
& A_{1}\left[u, v ; I_{T}(u, v)\right]= \\
& \quad \frac{4}{\pi}\left\{1-\left[\frac{\left|F_{R}(u, v)\right|^{2}+\left|F_{S}(u, v)\right|^{2}-I_{T}(u, v)}{2\left|F_{R}(u, v)\right|\left|F_{S}(u, v)\right|}\right]^{2}\right\}^{1 / 2} .
\end{aligned}
$$

With the following threshold function, ${ }^{9}$

$$
I_{T}(u, v)=\left|F_{R}(u, v)\right|^{2}+\left|F_{S}(u, v)\right|^{2},
$$

the intraclass terms of Eq. (2) responsible of the selfcorrelation terms at the output plane are removed. This threshold function can be obtained by evaluation of the intensity of the Fourier transform of the scene and the reference separately.

Now Eq. (5) becomes

$$
\begin{aligned}
I_{b}(u, v)= & \frac{4}{\pi} \sum_{n=0}^{\infty} \frac{(-1)^{n}}{2 n+1} \cos \left\{( 2 n + 1 ) \left[2 \pi\left(x_{0} u+y_{0} v\right)\right.\right. \\
& \left.\left.+\phi_{R}(u, v)-\phi_{S}(u, v)\right]\right\} .
\end{aligned}
$$

We can consider only the first term of the previous expansion, since the remaining terms can be neglected, owing to their low energy:

$$
I_{b, 1}(u, v) \propto \cos \left[2 \pi\left(x_{0} u+y_{0} v\right)+\phi_{R}(u, v)-\phi_{S}(u, v)\right] .
$$

When the reference matches the scene $\left(f_{R}=f_{S}\right)$ in the correlation plane, we get

$$
c(x, y) \propto \delta\left(x-x_{0}, y-y_{0}\right)+\delta\left(x+x_{0}, y+y_{0}\right) .
$$

Note that the usual diffraction spot in the center of the correlation plane is not present, although there are still two cross-correlation terms not centered at the origin.

\section{On-Axis Joint Transform Correlator}

To design an on-axis JTC, we propose to compute the complex transmittance

$$
\exp \left\{i\left[\phi_{R}(u, v)-\phi_{S}(u, v)\right]\right\}
$$

instead of the cosine term of relation (10). The Fourier transform of relation (12) gives a single centered detection peak,

$$
c(x, y) \propto \delta(x, y) .
$$

Because there is no linear phase term $\left[2 \pi\left(x_{0} u+\right.\right.$ $\left.\left.y_{0} v\right)\right]$ in the transmittance in relation (12), the scene and the reference should be displayed superimposed on the LCD. Therefore both the input and the correlation plane images are on axis, and the spacebandwidth product of the CCD camera is optimized.

We next describe how to obtain the transmittance in relation (12). First, we introduce the scene and the reference centered at the input plane,

$$
f_{R}(x, y)+f_{S}(x, y)
$$

so, after a Fourier transform, we obtain the following JPS:

$$
\begin{aligned}
I_{C}(u, v)= & \left|F_{R}(u, v)\right|^{2}+\left|F_{S}(u, v)\right|^{2}+2\left|F_{R}(u, v)\right| \\
& \times\left|F_{S}(u, v)\right| \cos \left[\phi_{R}(u, v)-\phi_{S}(u, v)\right],
\end{aligned}
$$

which can be registered by the camera and stored in the computer memory. If we again display the scene and the reference centered at the input plane but with a phase shift of $\pi / 2$ between them,

$$
f_{R}(x, y)+f_{S}(x, y) \exp (i \pi / 2),
$$

the JPS will be

$$
\begin{aligned}
I_{S}(u, v)= & \left|F_{R}(u, v)\right|^{2}+\left|F_{S}(u, v)\right|^{2}+2\left|F_{R}(u, v)\right| \\
& \times\left|F_{S}(u, v)\right| \cos \left[\phi_{R}(u, v)-\phi_{S}(u, v)-\pi / 2\right] \\
= & \left|F_{R}(u, v)\right|^{2}+\left|F_{S}(u, v)\right|^{2}+2\left|F_{R}(u, v)\right| \\
& \times\left|F_{S}(u, v)\right| \sin \left[\phi_{R}(u, v)-\phi_{S}(u, v)\right] .
\end{aligned}
$$

It is possible to have a phase shift between two images by use of the modulation properties of the LCD's, which are described by its operating curves, as we further explain in Section 4.

We then define a new function that combines the 


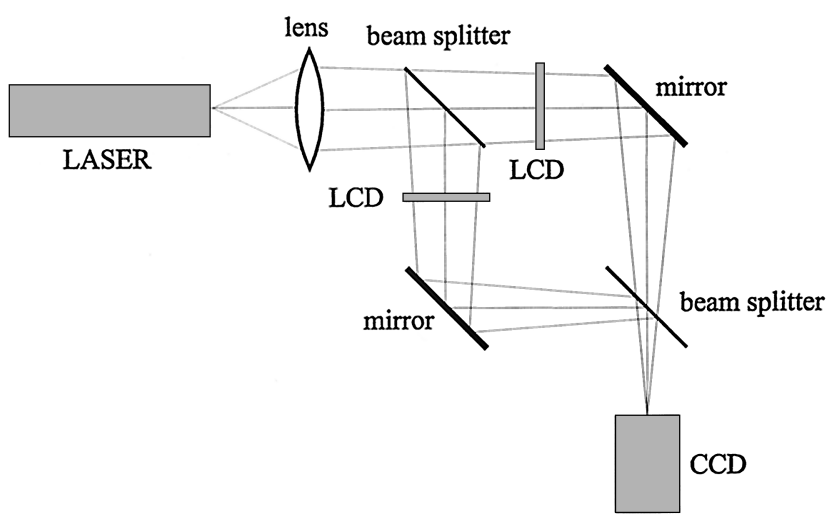

Fig. 2. Optical setup.

results of binarizing $I_{C}$ and $I_{S}, I_{q}(u, v)=B\left[I_{C}(u, v)\right]+$ $i B\left[I_{S}(u, v)\right]$, i.e.,

$$
\begin{aligned}
& I_{q}(u, v)= \\
& \left\{\begin{array}{rll}
1+i & \text { if } I_{C}(u, v) \geq I_{T}(u, v), \quad I_{S}(u, v) \geq I_{T}(u, v) \\
-1+i & \text { if } I_{C}(u, v)<I_{T}(u, v), \quad I_{S}(u, v) \geq I_{T}(u, v) \\
1-i & \text { if } I_{C}(u, v) \geq I_{T}(u, v), \quad I_{S}(u, v)<I_{T}(u, v) \\
-1-i & \text { if } I_{C}(u, v)<I_{T}(u, v), \quad I_{S}(u, v)<I_{T}(u, v)
\end{array}\right.
\end{aligned}
$$

If we use the threshold function of Eq. (8) and follow the mathematical procedure of Section 2 , the distribution $I_{q}(u, v)$ can be written as

$$
\begin{aligned}
I_{q}(u, v) \approx & \cos \left[\phi_{R}(u, v)-\phi_{S}(u, v)\right] \\
& +i \sin \left[\phi_{R}(u, v)-\phi_{S}(u, v)\right] \\
= & \exp \left\{i\left[\phi_{R}(u, v)-\phi_{S}(u, v)\right]\right\} .
\end{aligned}
$$

\section{Optical Setup}

To perform the whole process optically, we propose the architecture sketched in Fig. 2. This optical setup is similar to the nonconventional JTC, ${ }^{10,11}$ which basically aimed at obtaining a higher carrier fringe frequency. However, our goal is different, since we introduce the scene and the reference super-

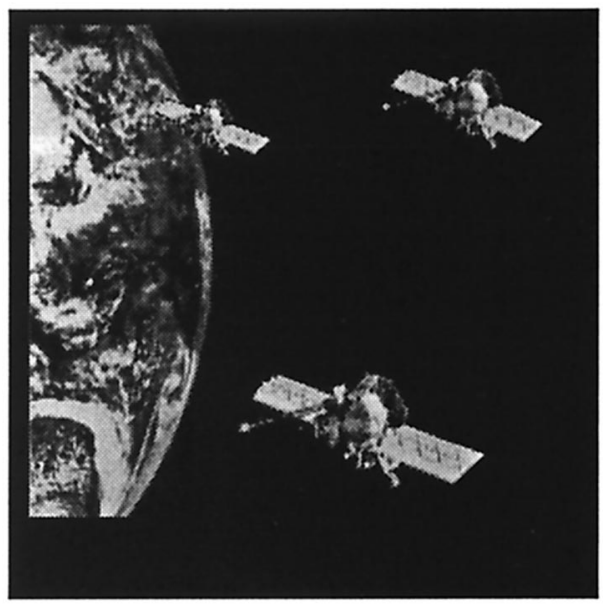

Fig. 3. Image used as scene.

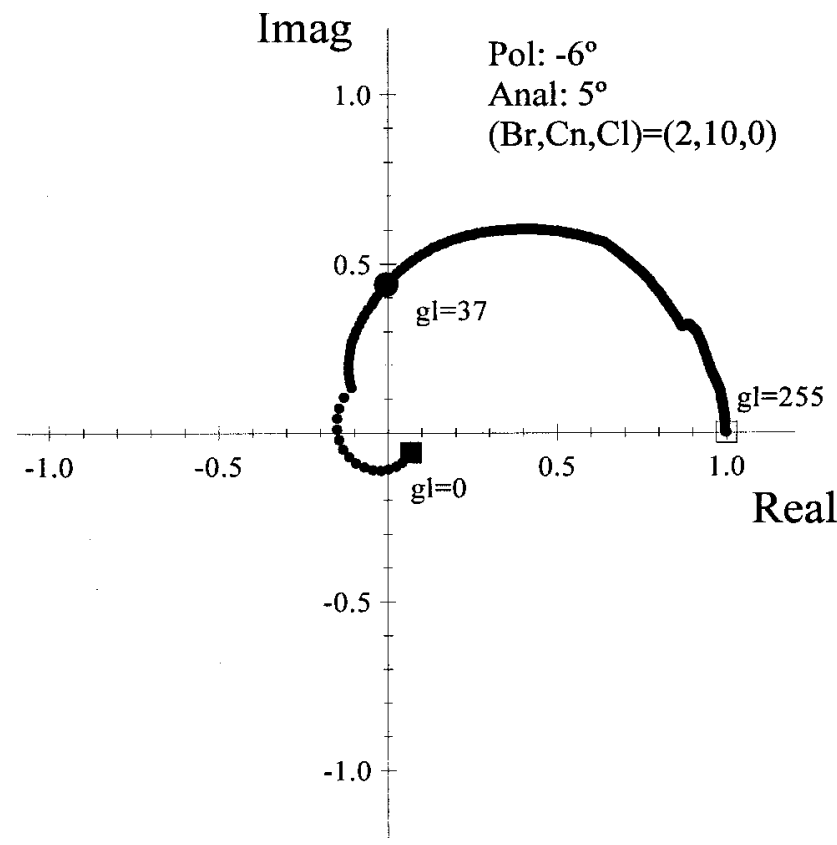

Fig. 4. High-contrast configuration.

imposed at the input plane to achieve a single centered correlation as a result.

In our setup the images are displayed on LCD's that modulate both the phase and the amplitude of the transmitted light, depending on the applied voltage. That voltage is applied by a digitizer board, which transforms different gray levels of images on a computer in electrical signals. It is then necessary to obtain the operating curves of the panel, which give the relationship between the gray level of the input image and the amplitude and phase modulation. Depending on the orientation of the polarizer and the analyzer placed before and after the LCD and on the values of some potentiometer controls available on the videoprojector (brightness and contrast), different kinds of response are obtained.

First, the cosine and the sine distributions [relations (14) and (16)] should be displayed at the input plane. For that purpose two LCD's are required. In the first case both of them should have the same amplitude modulation response. In the second case they should

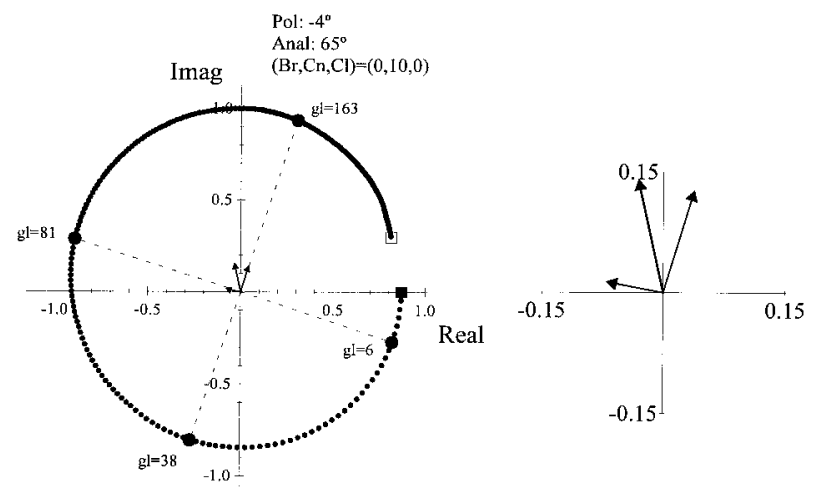

Fig. 5. Phase-mostly configuration and remaining transmittance for the four gray levels chosen. 
Table 1. Amplitude and Phase Modulation for the Gray Levels Chosen

\begin{tabular}{lcccc}
\hline & $\mathrm{gl}=6$ & $\mathrm{gl}=38$ & $\mathrm{gl}=81$ & $\mathrm{gl}=163$ \\
\hline Transm. (Ampl.) & 0.87 & 0.85 & 0.94 & 0.98 \\
Phase (rad) & $-0.10 \pi$ & $-0.60 \pi$ & $-1.10 \pi$ & $-1.60 \pi$ \\
\hline
\end{tabular}

modulate only the amplitude, but their operating curves should give a phase shift of $\pi / 2$ between them. In this way, after a Fourier transform, we obtain $I_{C}$ [Eq. (15)] and $I_{S}$ [Eq. (17)] by means of the CCD camera; so we can compute $I_{q}$ [Eq. (18)] digitally.

To perform the second Fourier transform and get the desired centered correlation plane, we need a single LCD with a configuration that gives the required four values of $I_{q}$. This can be achieved easily with a phase-only response, which has a constant transmittance and a wide range of phase variation.

\section{Experimental Procedure and Results}

The scene chosen for the experimental demonstration is shown in Fig. 3, and the verification of the method consists in the detection of each satellite. In the optical setup we used LCD's removed from an Epson Model VP100-PS videoprojector. With such devices we did not find the ideal aforementioned operating curves. Even so, it is still possible to carry out the whole process by use of different configurations.

To display the required distributions of relations (14) and (16), we chose a high-contrast configuration (Fig. 4) and a single LCD. With a single LCD the available dynamic range is generally reduced, but this is not our case, because the images we use never overlap. The high-contrast curve gives a high contrast between the gray level $\mathrm{gl}=255$ and $\mathrm{gl}=0$ and a phase
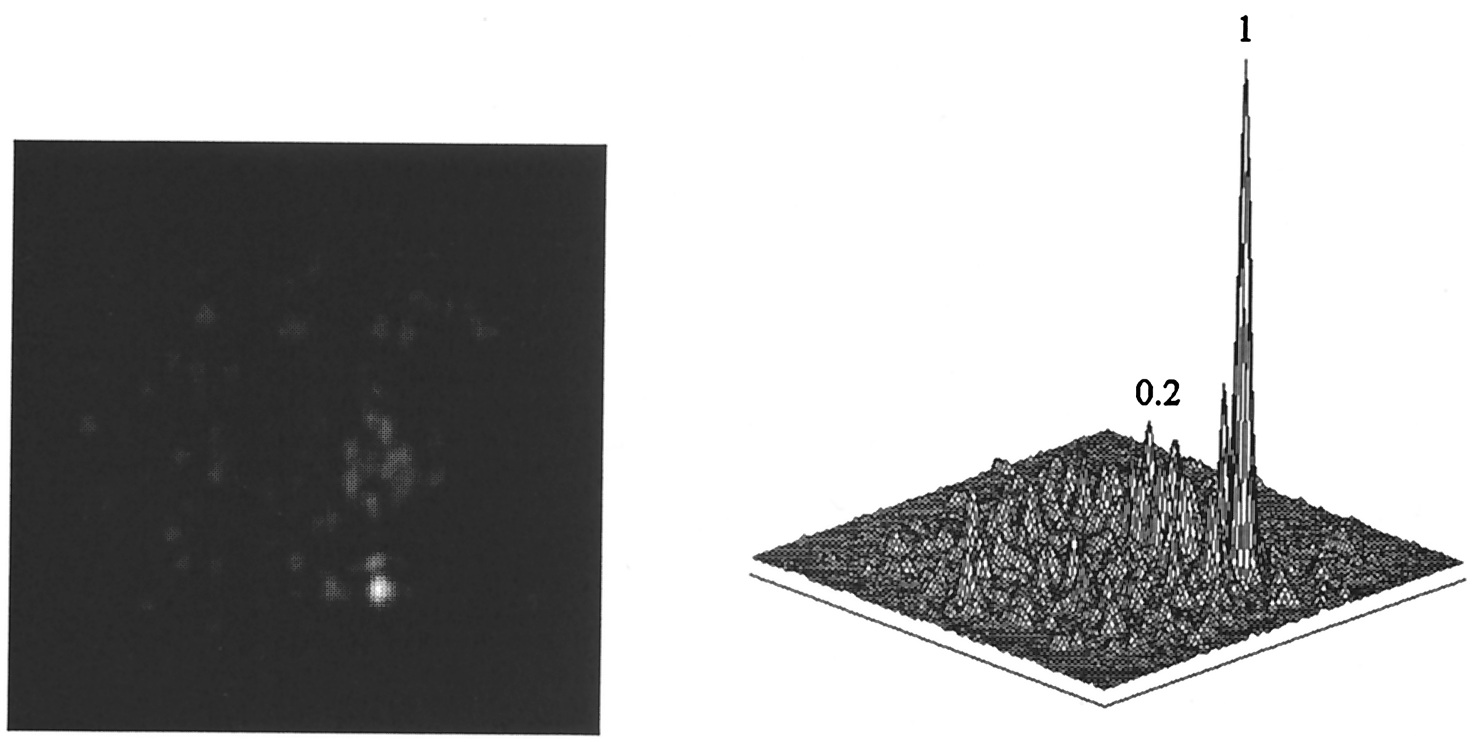

Fig. 6. Total correlation plane. Detection of the bottom satellite.
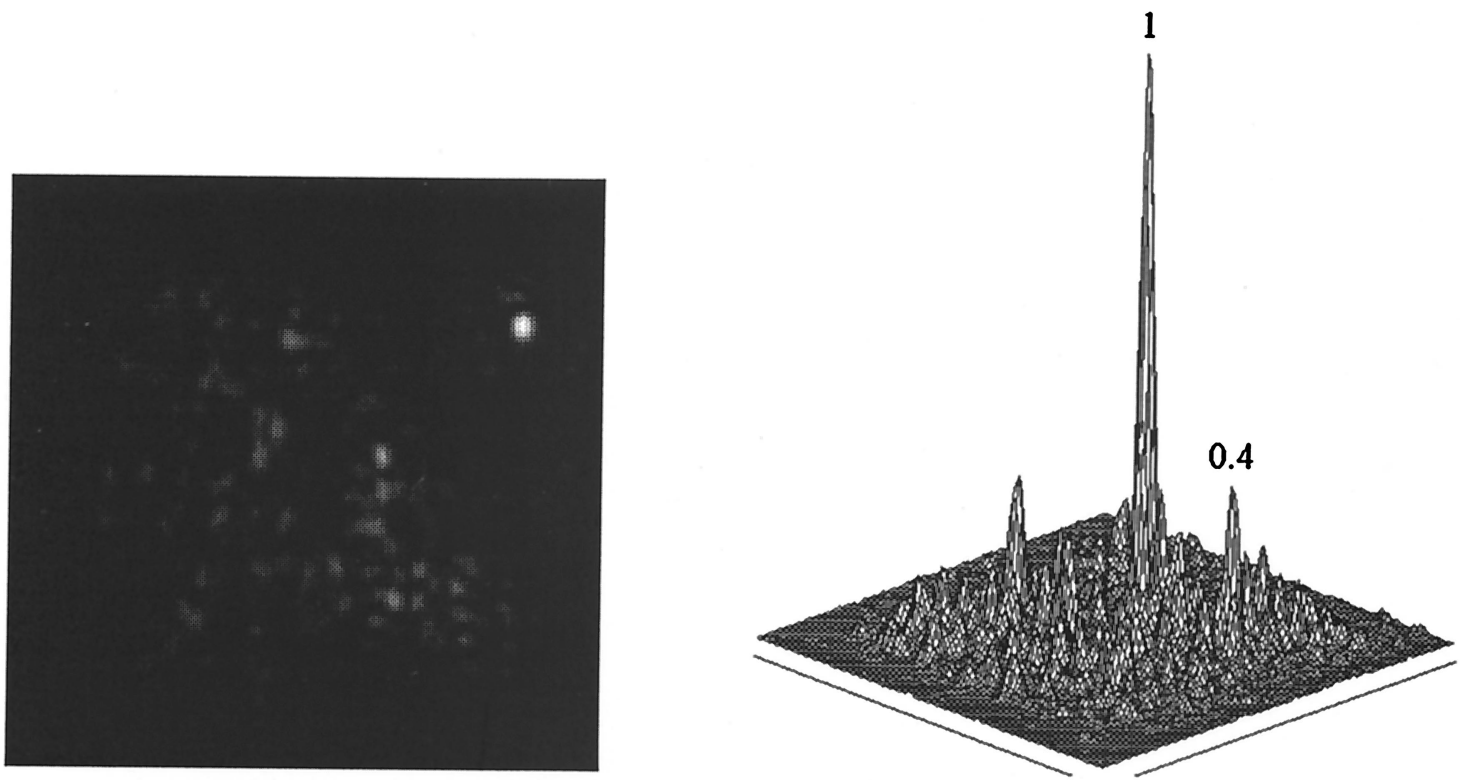

Fig. 7. Total correlation plane. Detection of the upper right-hand satellite. 

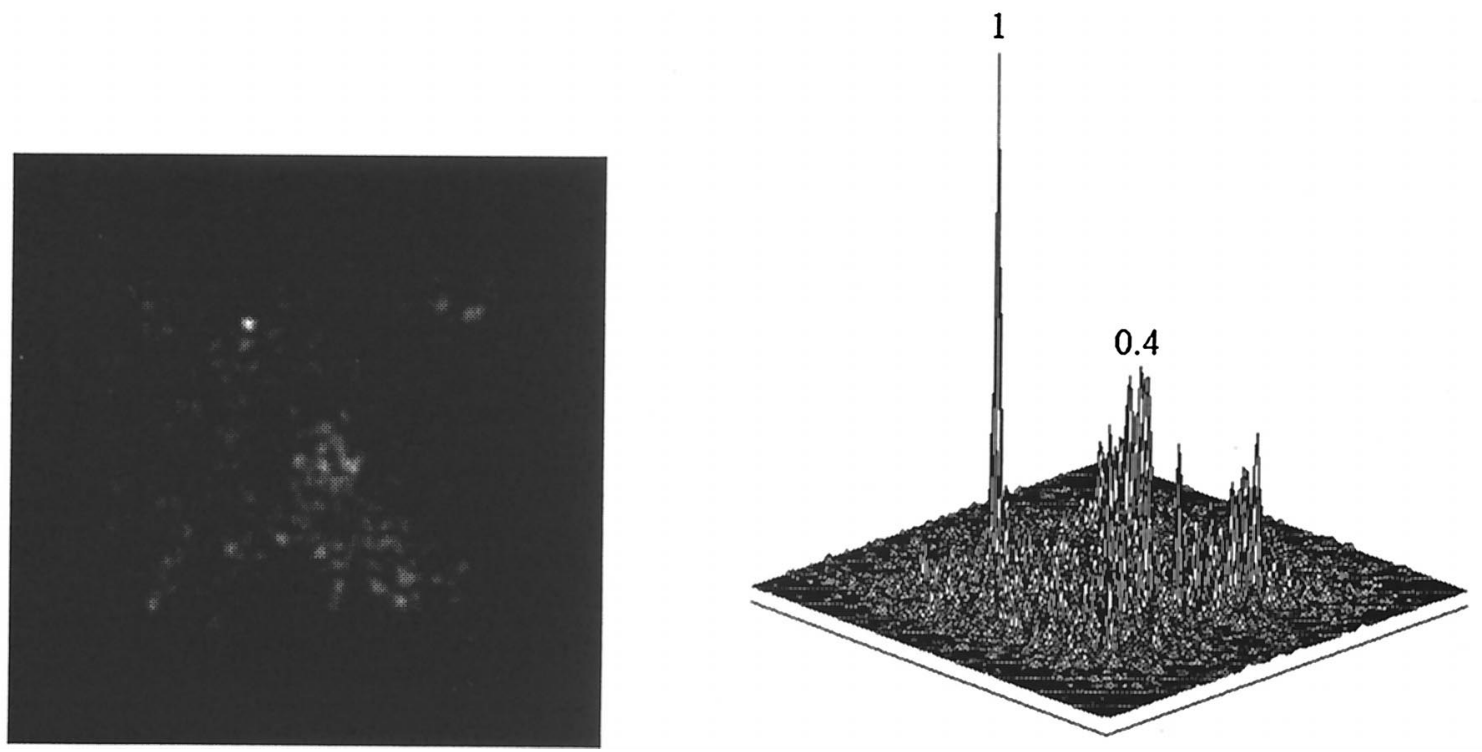

Fig. 8. Total correlation plane. Detection of the upper left-hand satellite.

shift between $\mathrm{gl}=255$ and $\mathrm{gl}=37$ of $\pi / 2$, as needed. This requires the input images to be binary. ${ }^{6}$

The scene and the references were binarized by application of a Sobel edge-detection filter and a threshold equal to their median value. First, $I_{C}$ [Eq. (15)] is obtained by displaying of both binary images with $\mathrm{gl}=255$ and $\mathrm{gl}=0$. When we obtain $I_{S}$ [Eq. (17)], the same binary reference is used and the scene is displayed with $\mathrm{gl}=37$ and $\mathrm{gl}=0$.

A phase-mostly configuration (Fig. 5) is used to display the $I_{q}$ [Eq. (18)] distribution. Four gray levels that have the same transmittance and with relative phase shift of $\pi / 2$ are required. In Table 1 the amplitude and the phase modulation of the values we chose are indicated. It can be seen that the whole transmittance is not null as wanted. The remaining amplitude is $\sim 0.14$, as shown in Fig. 5. Alternative sets of four gray values can also be used, giving similar results.

Finally, the correlation is obtained. Figures 6-8 show the correlation planes when each satellite is detected. The detection peaks are higher than the sidelobes present in the correlation planes, as shown in the three-dimensional plots. The central order should be null if the amplitude modulation of the four $I_{q}$ distribution values were the same; so the whole transmittance of the LCD was null. This is not possible for the operating curve we have. Nevertheless, there is a way to overcome this problem: We can compensate the remaining transmittance by assigning the required gray-level values to a certain area of the LCD without significant information. Moreover, the addressing of the LCD by the frame grabber is only approximately pixel by pixel, and therefore inaccurate gray values due to resampling are displayed, generating noise in the correlation plane.

\section{Conclusions}

We have proposed a JTC setup for obtaining a single centered correlation term. The use of this setup optimizes the space-bandwidth product of both the LCD and the CCD camera as well as the required light for the entire process, although it is necessary to have a good knowledge and exact control of the different optoelectronic devices involved. We have also carried out an experimental verification of the method, showing promising results, which surely can be improved by use of devices with greater resolution and dynamic range.

This paper was supported in part by Comisión Interministerial de Ciencia y Tecnología (CICYT) project TAP97-0454.

\section{References}

1. C. S. Weaver and J. W. Goodman, "A technique for optically convolving two function," Appl. Opt. 5, 1248-1249 (1966).

2. F. T. S. Yu and X. J. Lu, "A real time programmable joint transform correlator," Opt. Commun. 52, 10-16 (1984).

3. F. T. S. Yu, S. Jutamulia, T. W. Lin, and D. A. Gregory, "Adaptive real-time pattern recognition using a liquid crystal television based joint transform correlation," Appl. Opt. 26, 1370-1372 (1987).

4. Q. Tang and B. Javidi, "Technique for reducing the redundant and self-correlation terms in joint transform correlators," Appl. Opt. 32, 1911-1918 (1993).

5. G. Lu, Z. Zhang, S. Wu, and F. T. S. Yu, "Implementation of a non-zero-order joint-transform correlator by use of phaseshifting techniques," Appl. Opt. 36, 470-483 (1997).

6. E. Martín-Badosa, A. Carnicer, I. Juvells, and S. Vallmitjana, "Complex modulation characterization of liquid crystal devices by interferometric data correlation," Meas. Sci. Technol. 8, 764-772 (1997).

7. C. T. Li, S. Yin, and F. T. S. Yu, "Nonzero-order joint transform correlator," Opt. Eng. 37, 58-65 (1998).

8. B. Javidi, "Nonlinear correlation joint transform correlation," Appl. Opt. 28, 2358-2367 (1989).

9. B. Javidi, J. Wang, and Q. Tang, "Multiple-object binary joint transform correlation using multiple level threshold crossing," Appl. Opt. 30, 4234-4244 (1991).

10. T. C. Lee, J. Rebholz, and P. Tamura, "Dual-axis joint-Fouriertransform correlator," Opt. Lett. 4, 121-123 (1979).

11. F. T. S. Yu, C. H. Zhang, Y. Jin, and D. A. Gregory, "Nonconventional joint-transform correlator," Opt. Lett. 14, 922-924 (1989). 